\title{
استخدام حدائق السطح كاحد تطبيقات شبكات البنية التحتية الخضراء لتحقيق استدامة المدن الجديدة
}

$$
\text { احمد اسامة هارون }
$$

مدرس بقسم الهندسة المعمارية

كلية الهندسة - الجامعة الحديثة للتكنولوجيا والمعلومات archharon@gmail.com

الكلمات المفتاحية :

حدائق الاسطح، شبكات البنية التحتية الخضراء، استدامة، السمدن الجديدة الاسبط

\section{1. شبكات البنية التحتية الخضراء:}

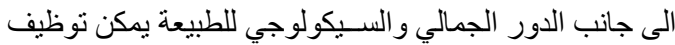

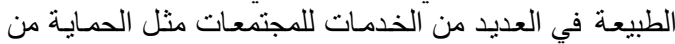

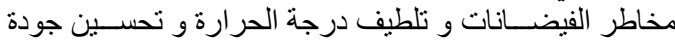

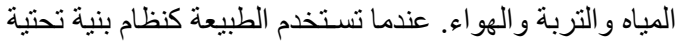

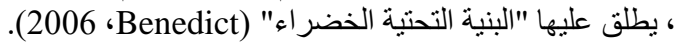

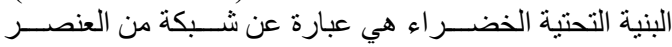

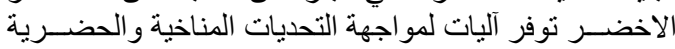

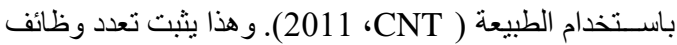

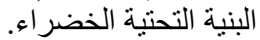

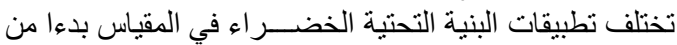

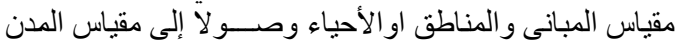

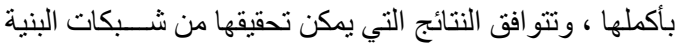

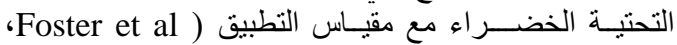

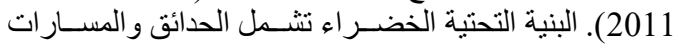

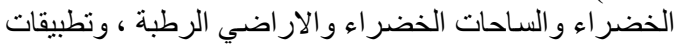

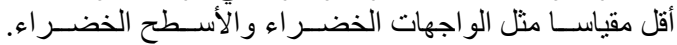

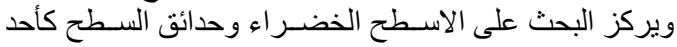
الحلول لاسطح المباني في مصر وما لاعلعانيه من مشكلات.

\section{2. حائق الاسطح :}

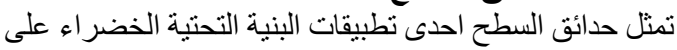

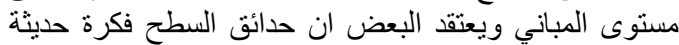

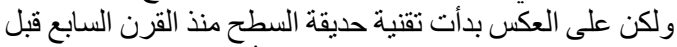

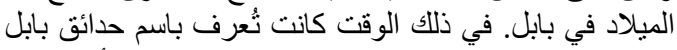

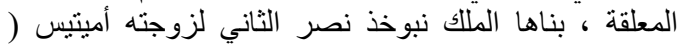

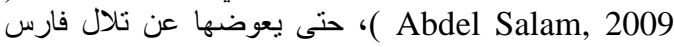

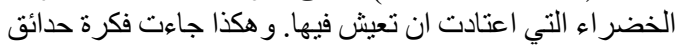

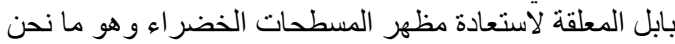
في امس الحاجة له الان خاصة مع نقص نصيب الفرد من من لفن

\author{
زينب فيصل عبد القادر \\ استاذ مساعد بقسم الهندسة المعمارية \\ كلية الهندسة ببنها - جامعة بنها
}

zeinab_feisal@hotmail.com

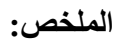

في ظل التغيرات المناخية الحالية تسعى العديد من الدول الى الى

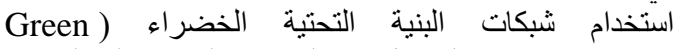

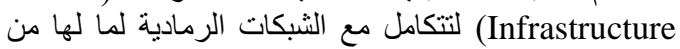

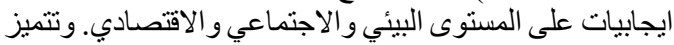

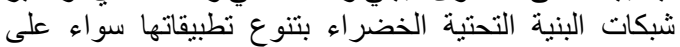

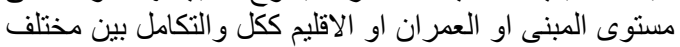

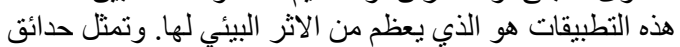

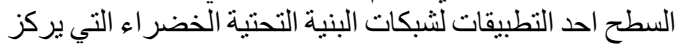

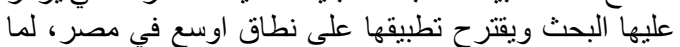

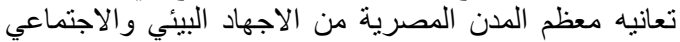
و الاقتصادي. تعتبر مدن اقليم القاهرة الكبرى من اكثر المدن في مصر التي لقدي

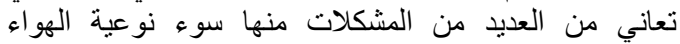

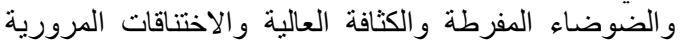

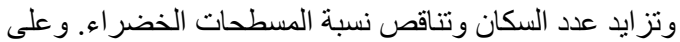

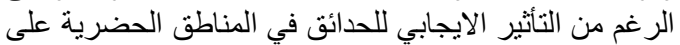

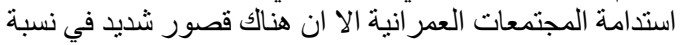

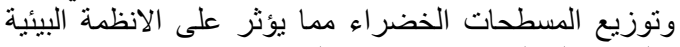

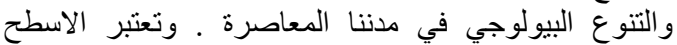

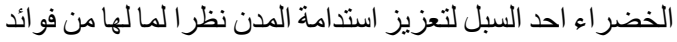

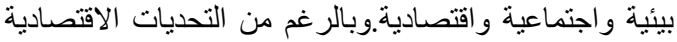

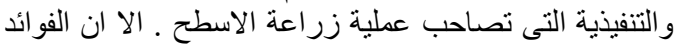
المباثرة والغير مباشرة اقتصاديا واجتماعيا وبيئيا اكبر واوسع الأنع

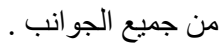
ويهدف البحث الى توضيح التحديات التشريعية و والتنفيذية لحدائق الاسطح في المدن الجديدة بالأضافة الى الى الفو ائد الناتجة عن تصميم حدائق الاسطح على الوحد الجيدة بالات السكنية و على المدينة

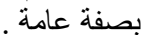
ويتناول البحث بالدر اسة استخدام تقنية زر اعة حدائق الاسطح

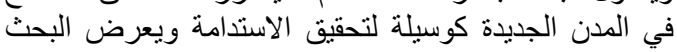

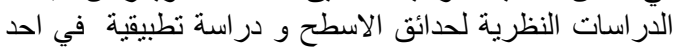

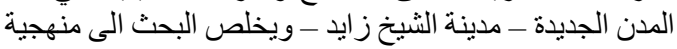
تطبيقية لتنفيذ هذا النموذج في العديد من الددن الجديدة. 
حيقة السطح الغير المكثفة/ الممتدة Extensive Roof Garden هي عبارة عن أسطح خضر اء ممتدة النطاق ، لها أعماق أقل من التح

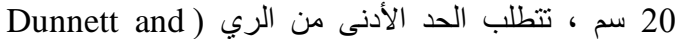
زراعه Kingsbury, 2004 وفي هذا النوع من الأسطح بمكننا

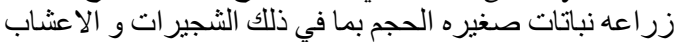

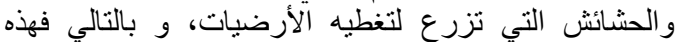

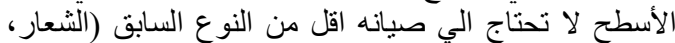

(2017

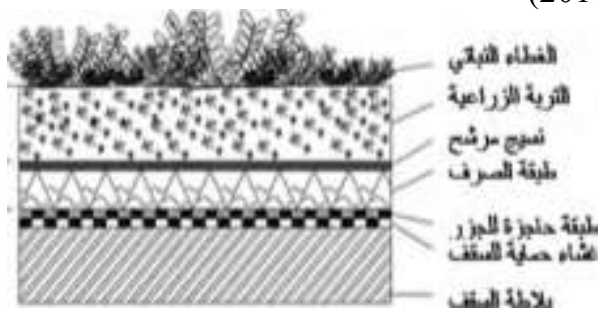

شكل 2 : طبقات حدائق السطح (الغير مكثفة/ الممتدة)

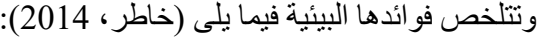
حماية سطح المبنى من العوامل الجئية الجوية المتغيرة.

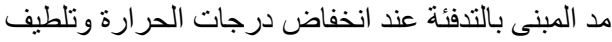

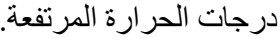

المساهمة فى استغلال مياه الأمطار والتقليل من نسبة

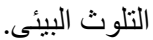

الامج بين حديقة السطح المكثفة والممتدة النطاق :

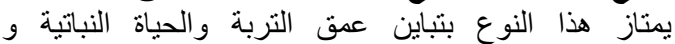
الإستخدامات الوظيفية (Mclntosh, 2010)

حائق السطح المثمرة Productive Green Roofs: و هي عبارة عن زر اعة حدائق أسطح المباني بالخضر و الفئن الفاكهة

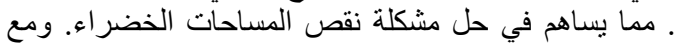

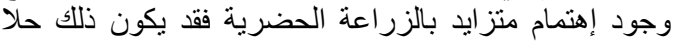
ايجابيا في حال تعميمه علي المباني بمختلف الإستعمالات

(خاطر، 2014).

\section{3. حدائق السطح والاستدامة:}

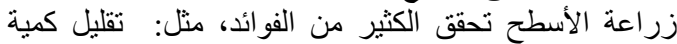

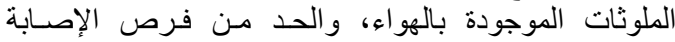

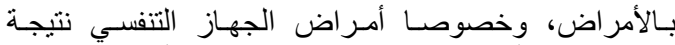

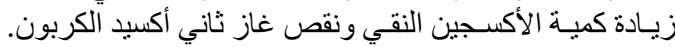

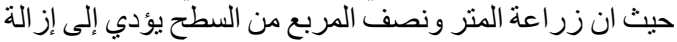

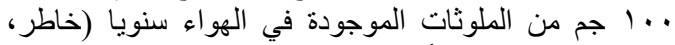

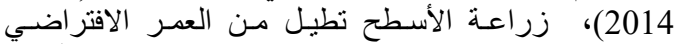

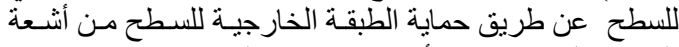

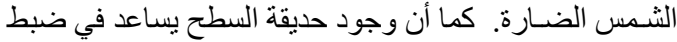

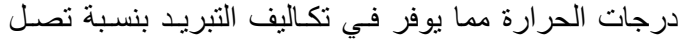

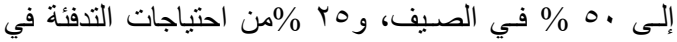
الثتاء (خاطر، 2014). ويوضح الثكل 3 دور حدائق السطح

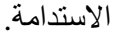

المسطحات الخضر اء في القاهرة الكبرى ليصل الى 0,33 م2

(Attia, 2009)

إن زراعة الحدائق على أسطح المبانى لا تقتصر على تحقيق

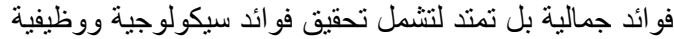

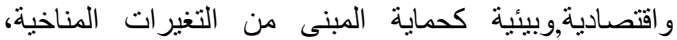

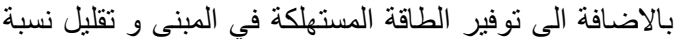

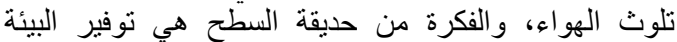
الطبيعية الجيدة للإنسان و المساعدة على التيل التز انه النفسى و الارتباط

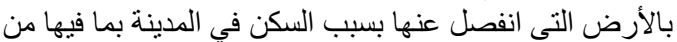

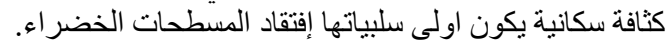

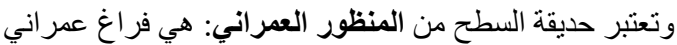

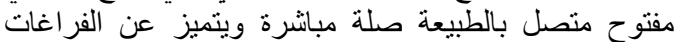

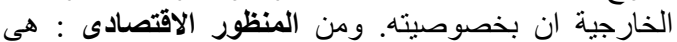

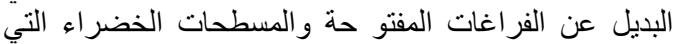

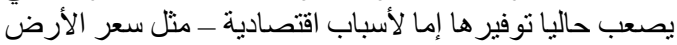

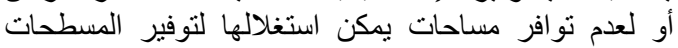

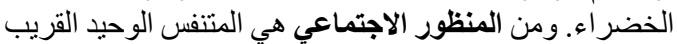

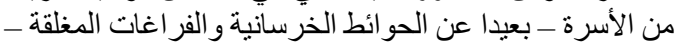
بالإضافة إلى كونها مكان للتجمعات و العلاقات الاجتماعية.

\section{ويمكن تصنيف حدائق السطح الى :}

حديقة السطح المكثفة Intensive Roof Garden

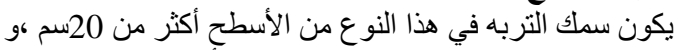

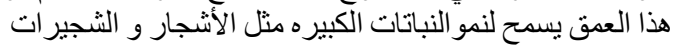

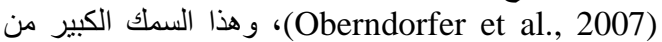

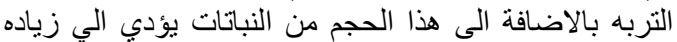

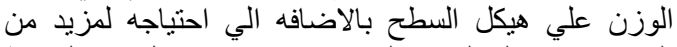

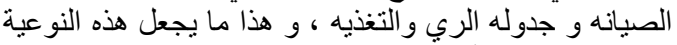
من حدائق السطح أكثر تكلفه (الثعار ، 2017).

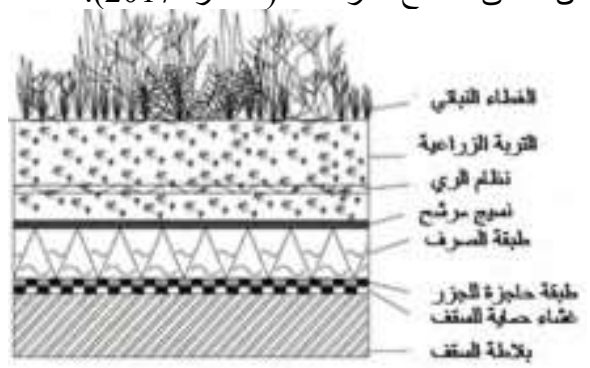

شكل 1 : مكونات/ طبقات حدائق السطح المكثفة

وتتلخص الفو ائد البيئية لهذا النوع من حدائق السطح فيما يلى الئى

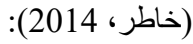

حماية السطح من درجات الحر ارة المنخفضة في الأماكن الباردة وتوفير مناطق مظللة فى سطح المبنى فى المناطق الاطق شديدة الحرارة. التقليل من تلوث الهواء حيث تقوم النباتات بامتصاص جزيئات الهو اء الملوثة. تجميع مياه الأمطار واستخدامها في عمليات الرى. 


\section{دور حائق السطح في تحقيق الاستدامة}

\begin{tabular}{|c|c|c|c|}
\hline الاستـامة الاقتصادية & الاستـامة الاجتماعية & الاستـامة البيئية & \\
\hline 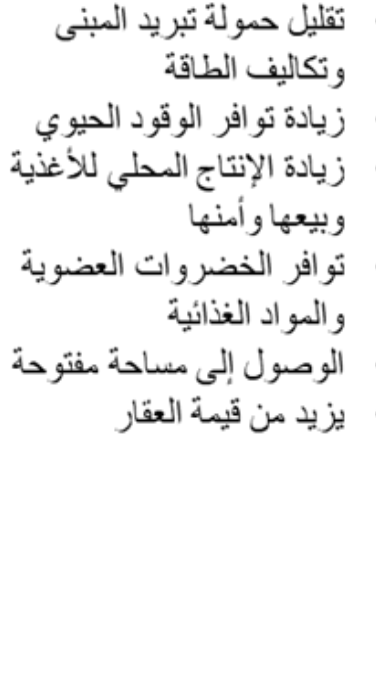 & 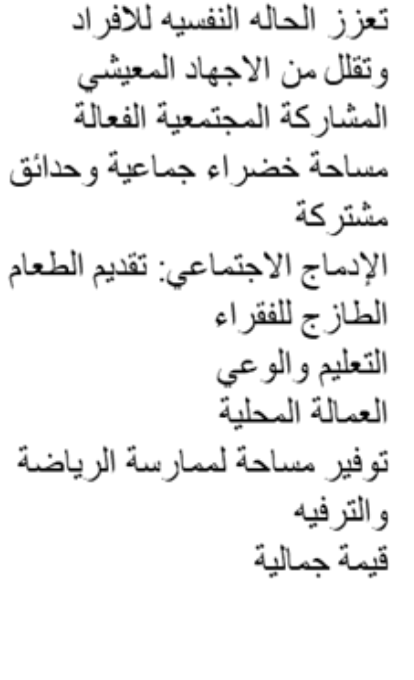 & 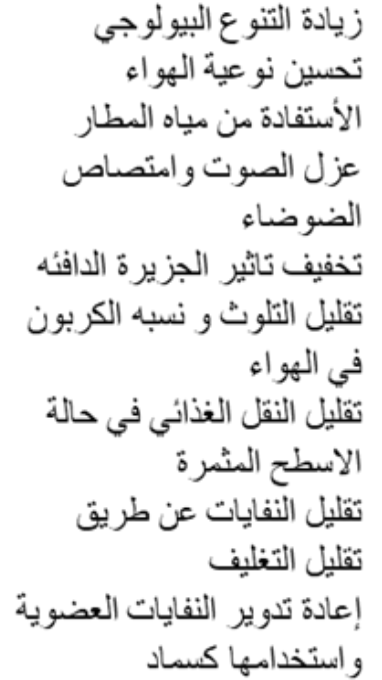 & $\begin{array}{l}\bullet \\
\bullet \\
\bullet \\
\bullet \\
\bullet\end{array}$ \\
\hline
\end{tabular}

شكل 3 : دور حدائق السطح في تحقيق الاستدامة على الجانب البيئي و الاجتماعي و الاقتصادي

المصدر (الباحث) المثن

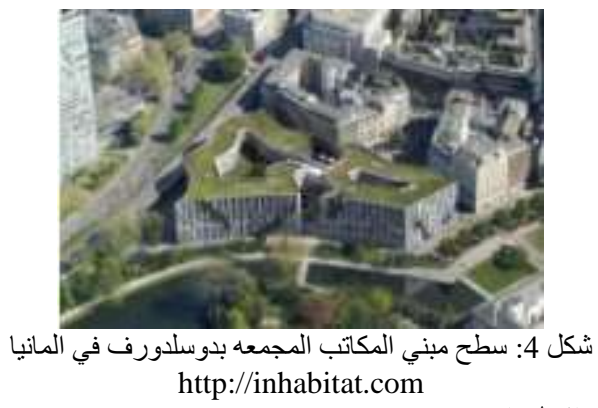

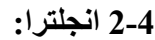

قام عمدة لندن Boris Johnson بنفيل أنفيل خطة لنشر الاسطح

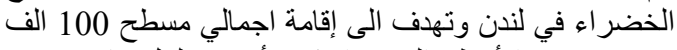

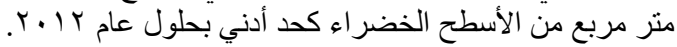

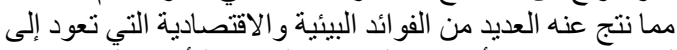

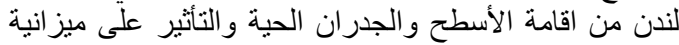

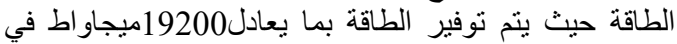
السنة، وتنقية الهواء بما بعادل 8256 طن من ثاني أكسيد

\section{4. حدائق السطح عالميا:}

عالميا لم تقتصر زراعة حدائق السطح على أسطح المبانى

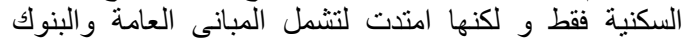

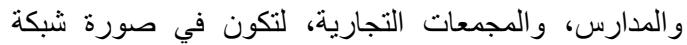

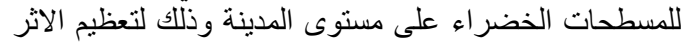

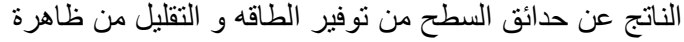
الجزر الحر اريه الي جانب الاستفادة من الجو انب البيائيه.

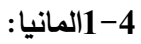

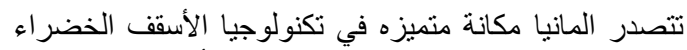

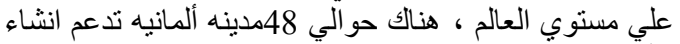

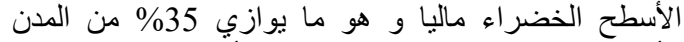

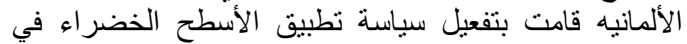

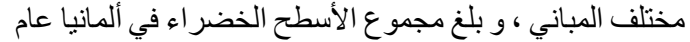

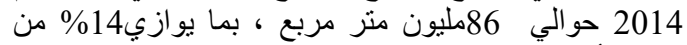

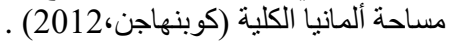


فيكون له العديد من الايجابيات من الجانب البيئي و الاجتماعي و الصحي و المناخي و الاقتصادي.

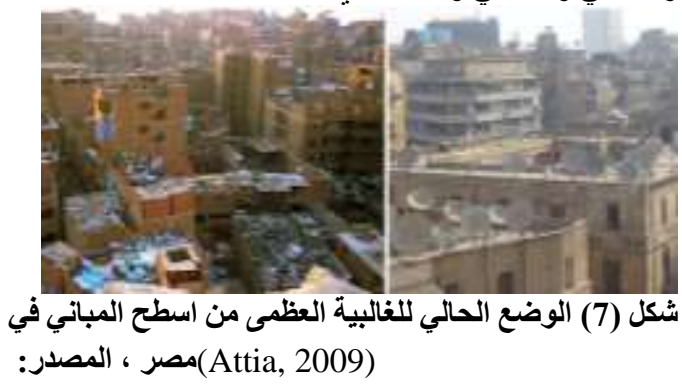

$$
\text { 6. حائق السطح - الحالة الدراسية: }
$$

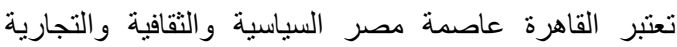

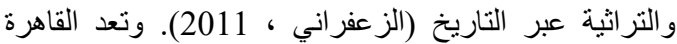
الكبري أكبر تجمع سكاني حضري في منطقيتي أفريقيا والثرق الثرق

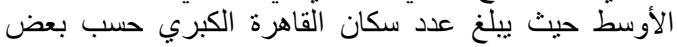

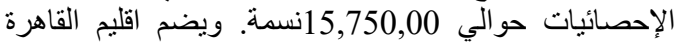

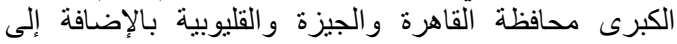

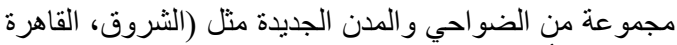
الجديدة، 6 أكتوبر ، الثيخ زايد الفي ، بدر ).

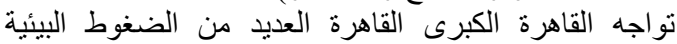

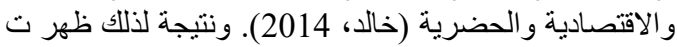

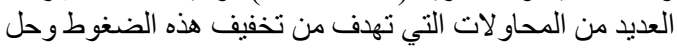

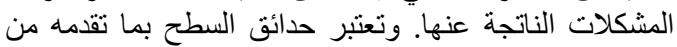

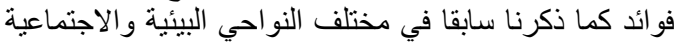

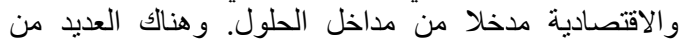
الابحاث والدراسات التي تناولت زراعة الاعلة الاسطح وخاصة في في

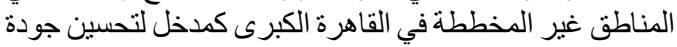

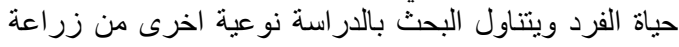

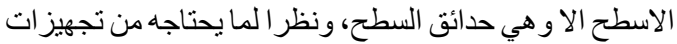

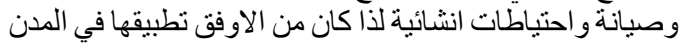

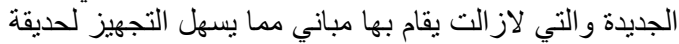

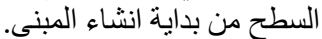

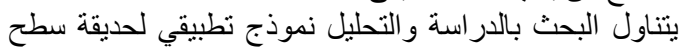
فوق احد المباني السكنية في مدينة الثيخ زايدة زليد، وتعتبر مدينة

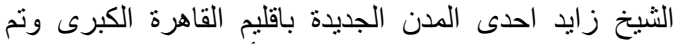

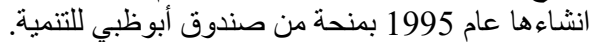

1-6 حديقة سطح بمدينة الثيخ زايد:

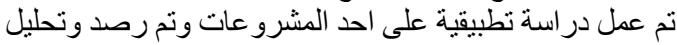
جميع المر احل التصميمية و الانثائية والتنفيذية لحدية التيقة السطح. وطبقا لأحتياجات السوق المصري والاني والعو امل الاقتصادية و البيئية

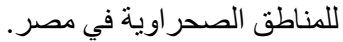

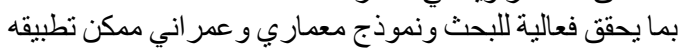

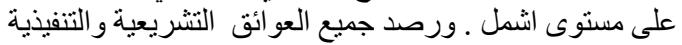
ووضعها في صورة مقترحات مستقبلية للبحث العلمي و التنفيذ في مر احل قادمة .
الكربون، بالاضافة الى القدرة على تخزين 80000 متر مكعب

من مياه الأمطار على مستوى السقف. لالفي

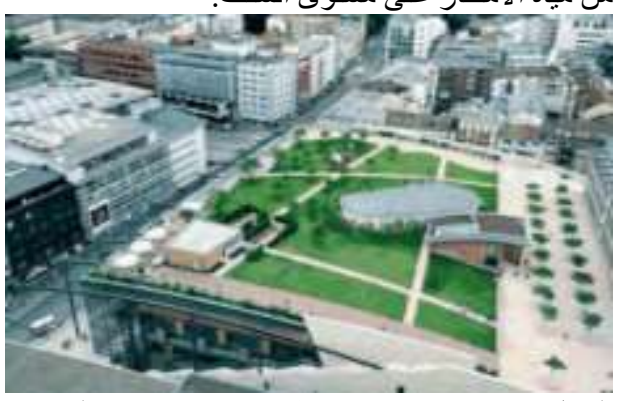

الثكل ( 5) يوضح حديقة سطح فندق Grange، لندن

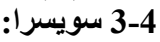

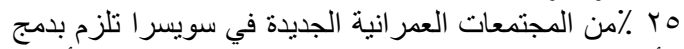

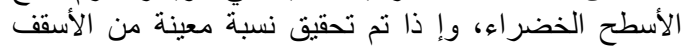

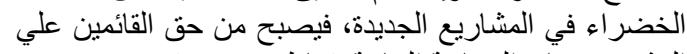

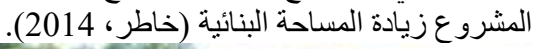

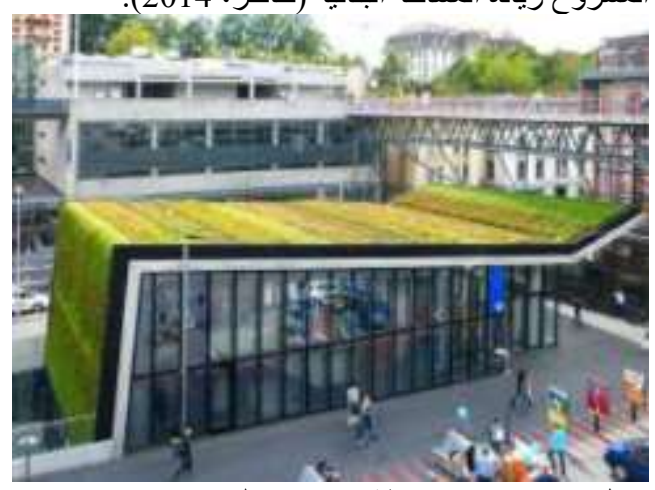

شكل (6) يوضح محطة مترو في لوزان، سويسر ا المصدر : / / / /

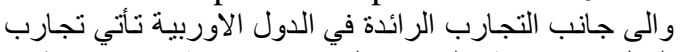

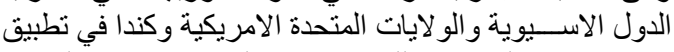

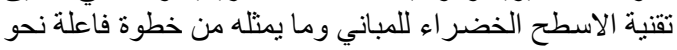

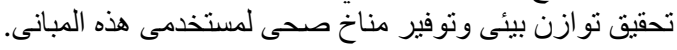

\section{5. اسطح المباني في مصر:}

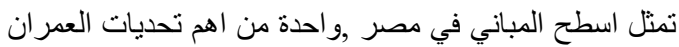

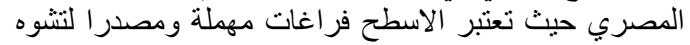

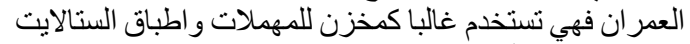

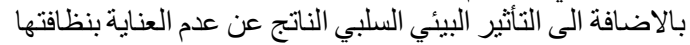

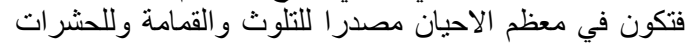

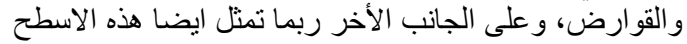

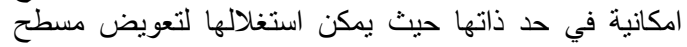

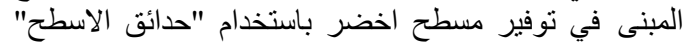


اولا: مرحلة العزل: العزئ

تعتبر مرحلة عزل السطح ضد العد الرطوبة من اهم المراحل

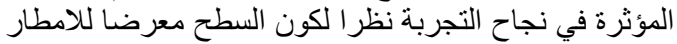

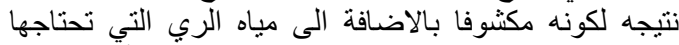

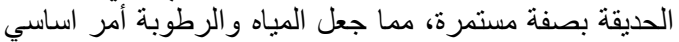

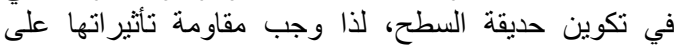

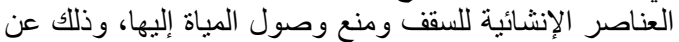

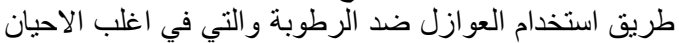

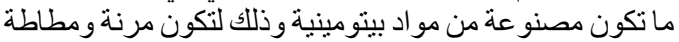
و غير قابلة للتشقق وسهلة التشكيل و اللصق و غير ونير منفذة للماء أو الأبخرة. وينبغي أن نتوافر في طبقات العزل الخصائص التالية (Luckett,2013) ـ القدرة على استيعاب الحركة الإنشائية العادية والإجهادات النانشئة عن مؤثرات الدور التئرات الحر ارية.

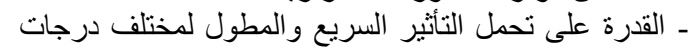

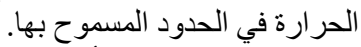

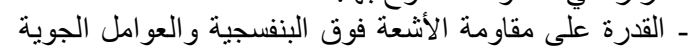

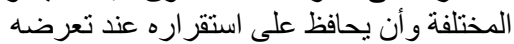

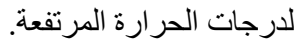

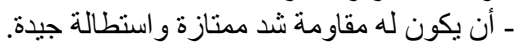
ـ أن يكون له مقاومة له عالية للصدمات مات و الخرق.

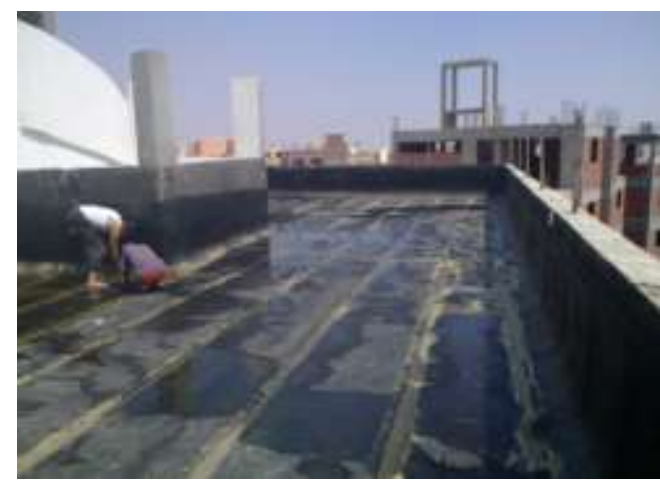

شكل (10) دراسة الحالة: مرحلة العزل Bitumen

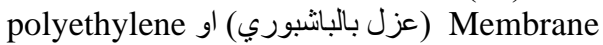
(المستخدم لعزل المسطحات المائيه)

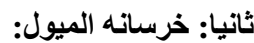

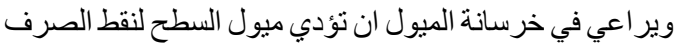

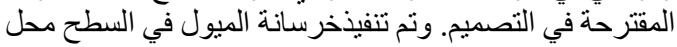
الدراسة بسمك يتر اوح بين 3 - 13 سم
والمبنى عبارة عن مبنى سكني بارتفاع 4 ادو ار مقام على قطعة

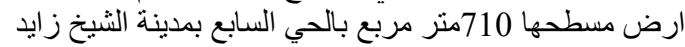

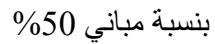

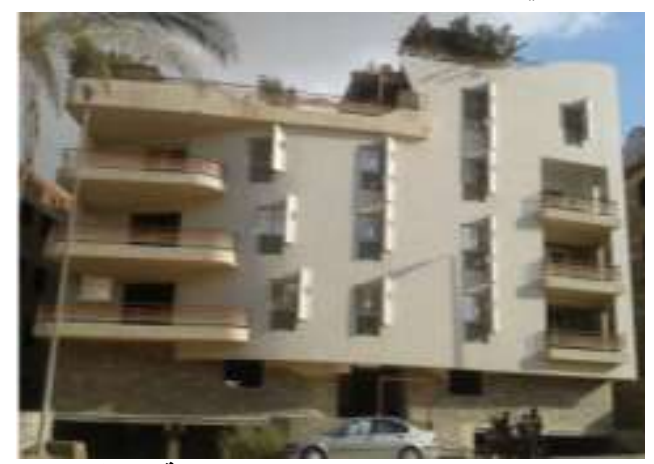

شكل (8) المبنى المقام عليه حليقة السطح

2-6 تصميم حديقة السطح: المبني المقات

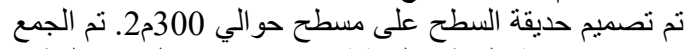

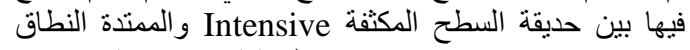
Extensive

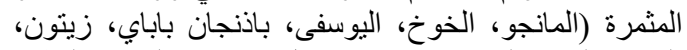

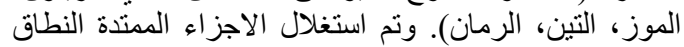

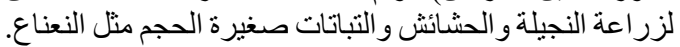

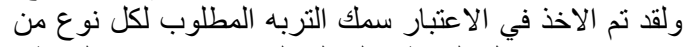

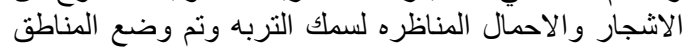
المخصصه للاشجار فوق الكمر ات وليس في منتصف البلاطه.

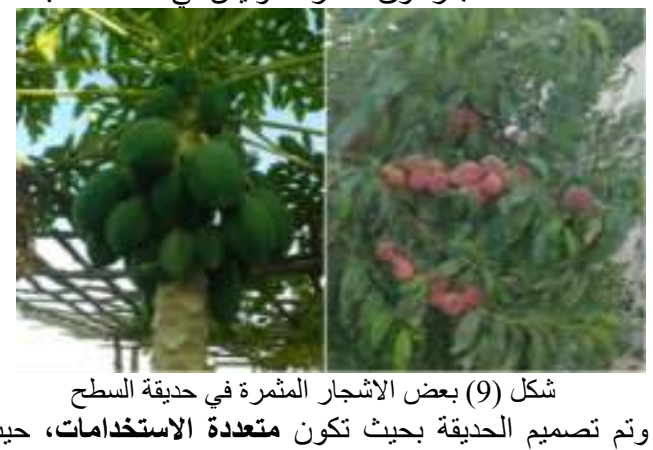

وتم تصميم الحديقة بحيث تكون متعددة الاستخدامات، حيث

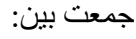
• • السطح المنتج للغذاء من خلال الاشجار المثرة

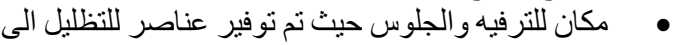
جانب الظل الناتج عن الاشجار

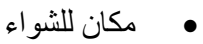
• مغطس صغير للاطفال للمول • مجمو عة من الخدمات صغلات

3-6 مراحل تنفيذ حديقة السطح : تتضمن عملية تنفيذ حديقة السطح مجموعة من المراحل: 


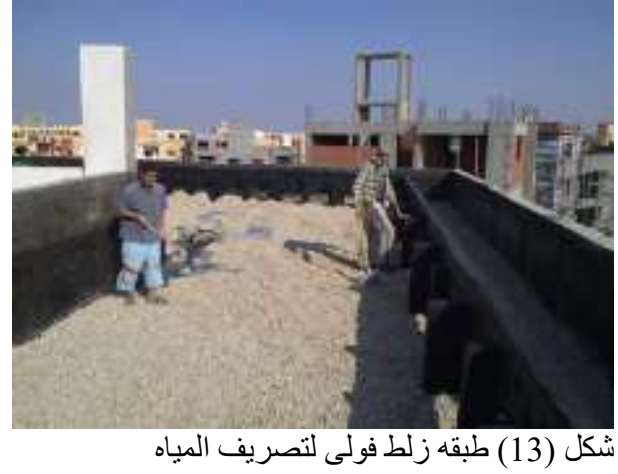

خامسا: طبقة النسيج المرشح: و هي الطبقة التي تكون اعلى طبقة التصني التصريف و ويعمل كفلتر لعدم

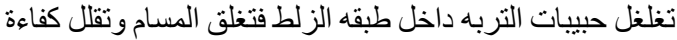

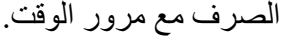

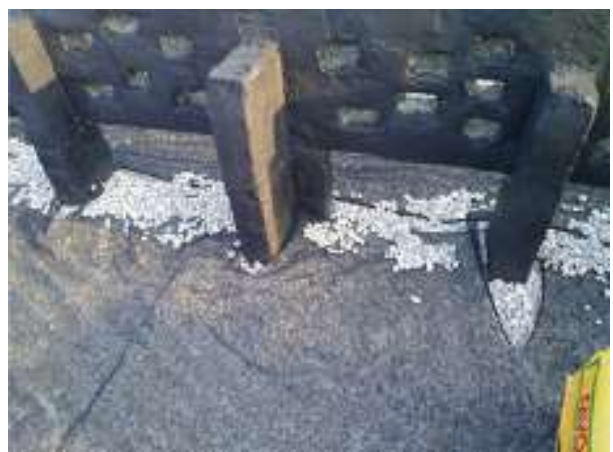

شكل (14) طبقة الثير ام (نسيج مرشح) سادسا: طبقة عزل الحرارة:

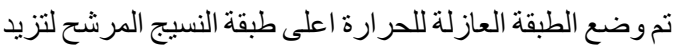

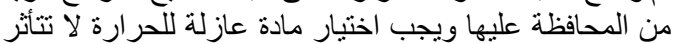

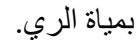

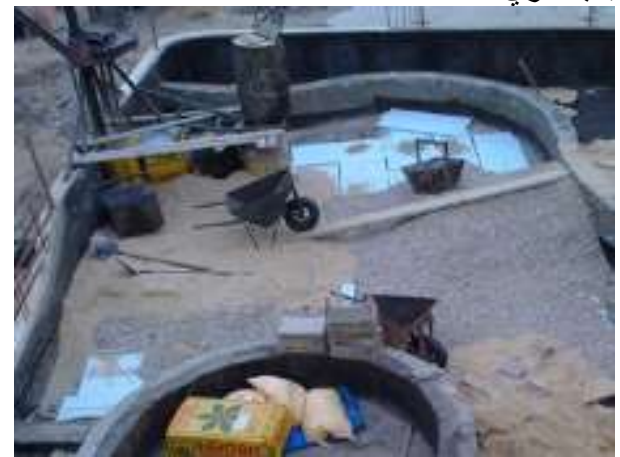

شكل (15) عازل حر ارة للتربه سمك اقل من 20 سم

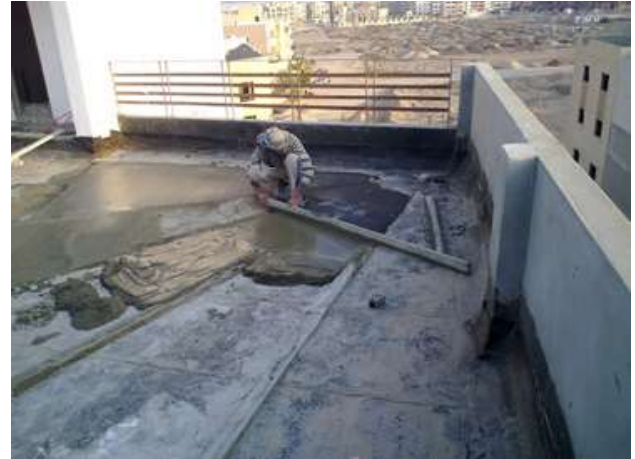

شكل (11) مرحلة تنفيذ خرسانة الميول

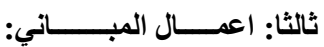

ويتم تحديد اعمال المباني المطلوب تلفئ تنفيذها طبقا للتصميم

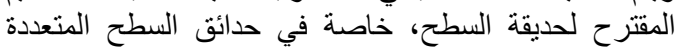

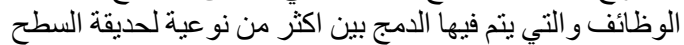

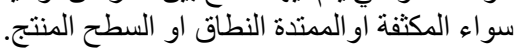

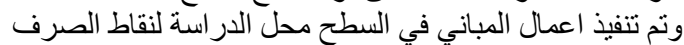

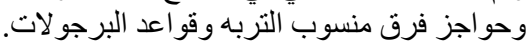

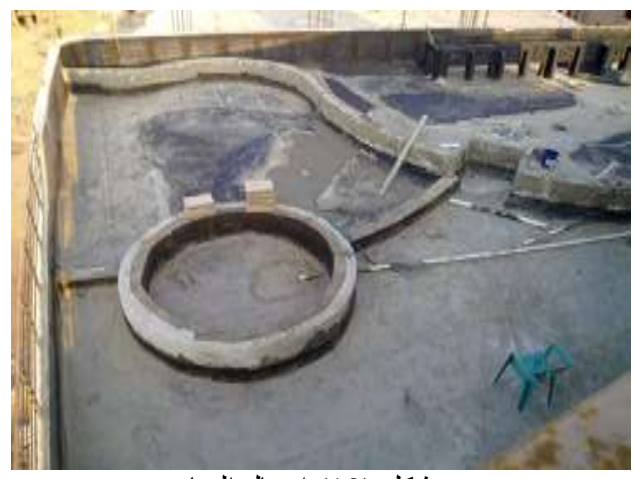

$$
\text { شكل (12) اعمال المباني }
$$

رابعا: طبقة تصريف المياه:

طبقة تصريف المياه الزائدة من الأحواض ومن الهن المسطحح

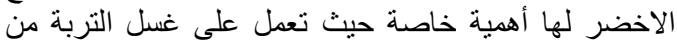

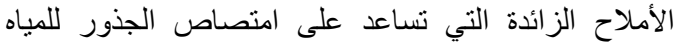

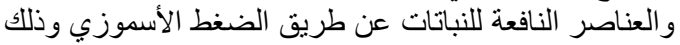

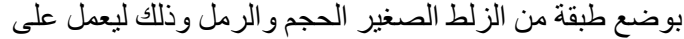

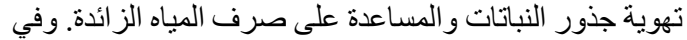

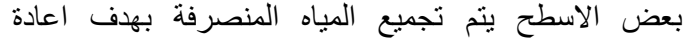
استخدامها في الري مرة اخرى. 
ينم في هذه المرحلة زر اعة النباتات المختلفة في السطح طبقا

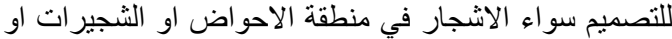

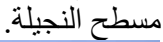

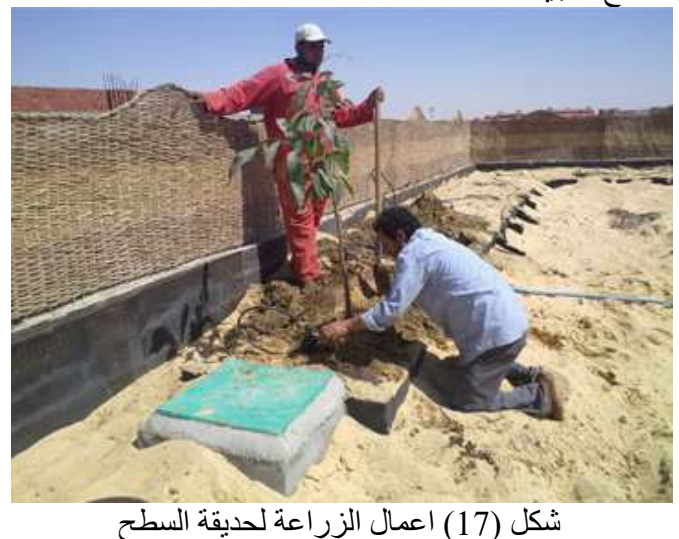

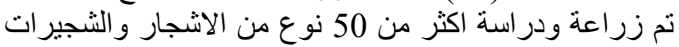

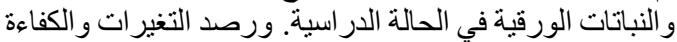

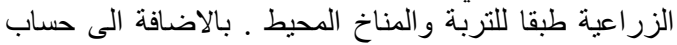
تكلف الصيانة والعائد الاقتصادي والمناخ لكل نوع من انو الاع النباتات .

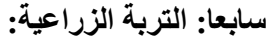
ويفضل في حالة زر اعة الاسطح ان يتم استخدام أنو اع من التربة

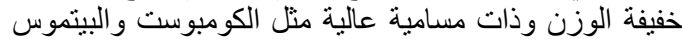
لتسعيل عمليات النقل بالاضافة الى تقليل الاحمال على على الاسطح

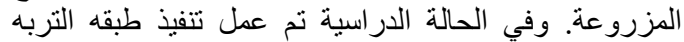
الزراعبه بالسمك المطلوب 20 سم للنجيله للشجير ات،60:80 سم للاشجار

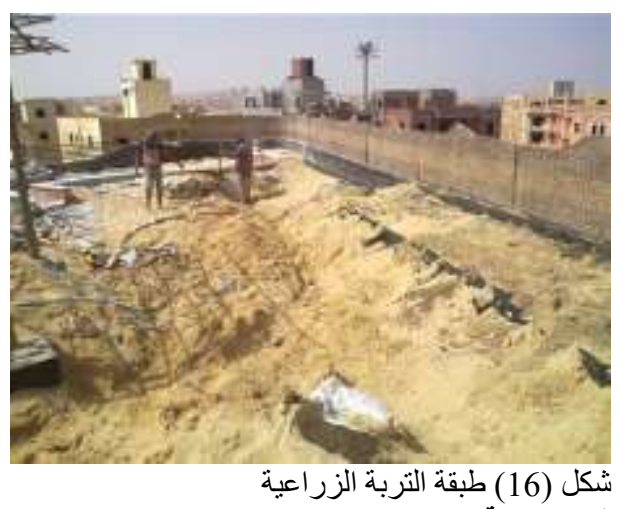
ثامنا: شبكة الري:

يفضل في هذه النوعية من الحدائق "حدائق السطح" استخدام

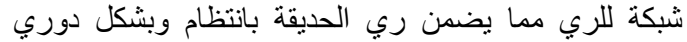

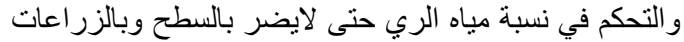
ويقلل الاعتماد على العنصر البشري.

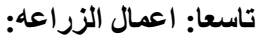

جدول (1) يوضح النباتات والاشجار المزروعة

\begin{tabular}{|c|c|c|c|}
\hline ملاحظات & 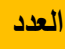 & الانواع & التصنيف \\
\hline ت تم زراعتها على حدود المحيط الخارجي للسطح & 30 & 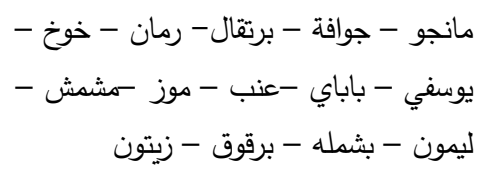 & الاشجار \\
\hline 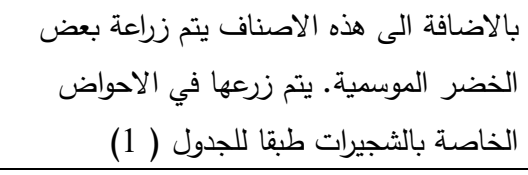 & 35 & 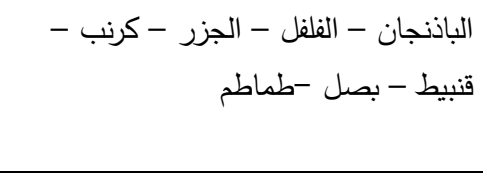 & 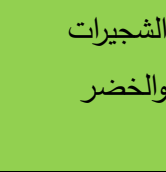 \\
\hline \multirow[t]{2}{*}{ يتم حصادها كل 15 يوم تقريبا } & 40 & زبدونس - شبت - كزبرة - ملوخية - & الانتاتات الورقي ذات \\
\hline & 5 & الياسمين - الفل - مسك الليل & الزينة الورود ونباتات \\
\hline
\end{tabular}



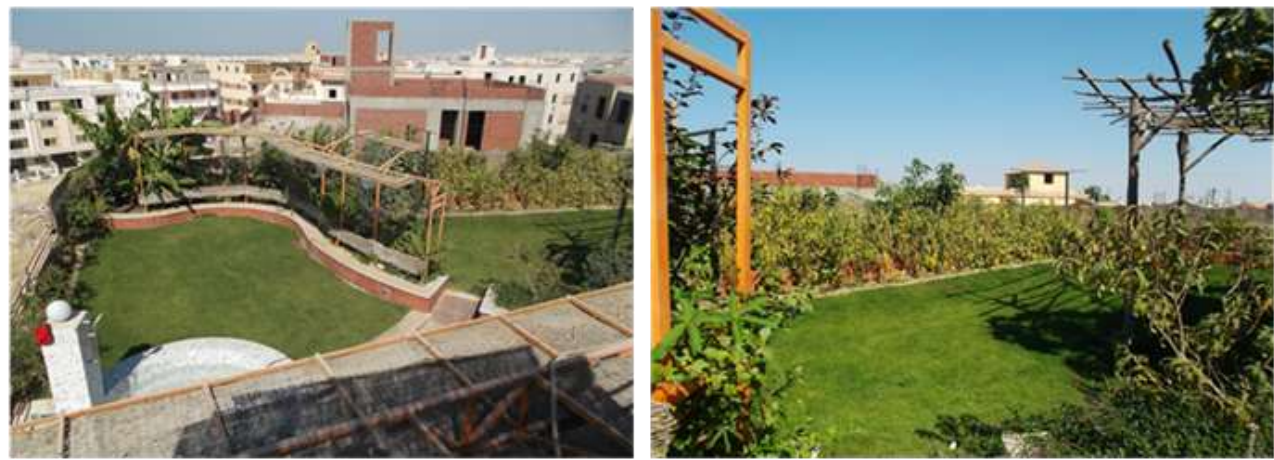

$$
\text { شكل (18) الثكل النهائي للسطح }
$$

- قد تبدو حديقة السطح مكلفة ولكن عند القيام بدراسة مقارنة

4-6 دور حديقة السطح في تحقيق الاستدامة: اقتصادية تبين ان تكلفة تشطيب السطح بالطريقة المعتادة اولا: الجانب البيئي: اعلى من عمل حديقة بالسطح كما يتضح من جدول 2

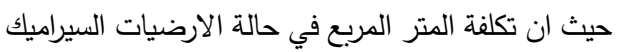
تصل الى 285ج وتكلفة المتر عند عمل الارضية مسطح

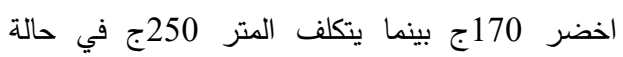
الاشجار والثجيرات، غير ان حديقة السطح تحتاج لصيانة ورعاية فيما بعد ولكن ما ينتج عنها من استفادة لايقارن بجهد وتكلفة الصيانة. ثالثا: الجانب الاجتماعي: وفرت حديقة السطح مكان للتجمعات آمن ويتمتع بالخصوصية وبعيد عن الضوضاء. بالاضافة ايضا الى توفير مساحة لممارسة الرياضة والترفيه.

- تقلل حديقة السطح من الانبعاثات الحراريه وتحد من استخدام المواد العازله للحراره اضافه الحد من التلوث والاتربه وزياده نسبة الاوكسجين في الجو.

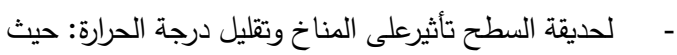
تم قياس درجة الحرارة على السطح الاخضر وسطح مبنى مجاور في اوقات مختلفة وكان متوسط فرق درجات الحرارة حوالي 5 درجات، وبقياس درجات الحرارة في الدور الاخير من المبنى والدور الاخير في مبنى مجاور كان الفرق في دان درجات الحرارة حوالي 7 درجات مئوية (الشعار ، 2017).

\section{ثانيا: الجانب الاقتصادي:} - تعتبر الحديقة منتجة لانواع مختلفة من الفاكهة والخضروات نتيجة زراعة الاشجار المثرة . توفر حديقة السطح العزل الجيد للدور الاخير مما يقلل من استهلاك الكهرباء في التكييف لوترب 
جدول 2 : مقارنة اقتصادية لتشطيب الاسطح المصدر: الباحث

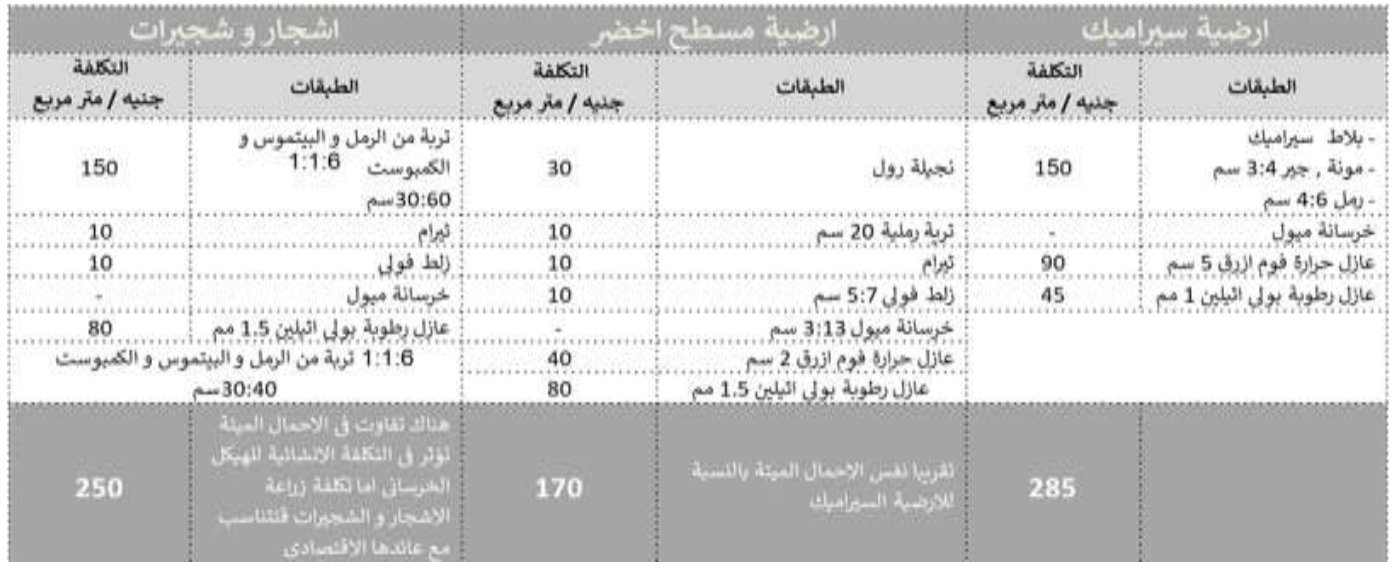

* النجيلة تحتاج ترية رملية فقط * الأشجار و الثجيرات بنصف الارتفاع تقرببا, التربة خليط من الرمل و البيتموس و

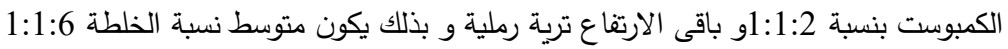

والقوارض والزواحف (العوائل الأساسية للعديدمن

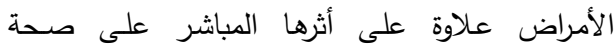
تقييم الحالة الاراسية بالتحليل البيئي SWAT:

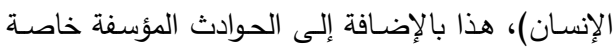

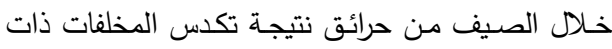
القابلية الكبيرة للاشتعال. اضافت الحديقة قيمة جمالية لمظهر العمران. ملكية المبنى تؤول الى عائلة واحدة مما سهل تطبيق الفكرة وساعد على الحفاظ على السطح وصيانته. - عن طريق حديقة السطح تم الاستفادة من مسطح غير مستغل فوق سطح المبنى لإنتاج الاحتياجات المنزلية

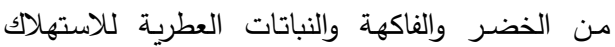
المنزلي ومن المكن في حالات اخرى يتم استغلالها لزيادة

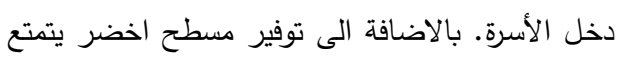
بالخصوصية والهدوء والامان.

المسطح الاخضر المضاف عن طريق حديقة السطح قد 7-2نقاط الضعف: عدم وجود حوافذ للملاك لتنفيذ حدائق السطح • مثل لماطل تخفيض فاتورة الكهرباء او المياة للمبنى المقام به حديقة سطح عدم وجود تشريع يحدد ملكية السطح لأحد الوحدات

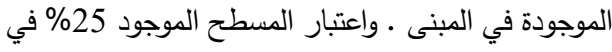

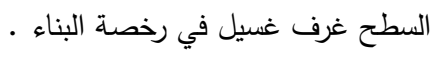
النقص الثديد في الإرشاد والكوادر الفنية المدربة للشرح ولتدريب الناس على تلك النظم وهي مسئولية معني بها لهاديا يؤدي بتعميم الفكرة الى تحسين الظروف البيئية وتقليل

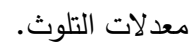
ادت حديقة السطح الى التخلص من الصسورة العامـة التي

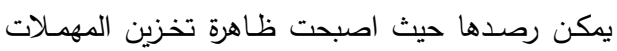

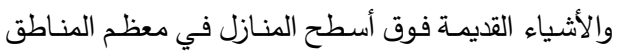

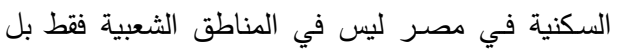
الراقية ايضا، وما يترتب على تلك الظـاهرة من أبعاد

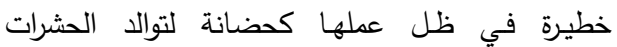


عدم حساب الاحمال الزائدة في حالة المباني قبل التنفيذ

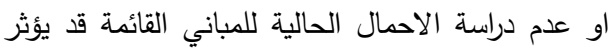
على الهيكل الانشائي للمبنى.

النتائج و التوصيات:

- ان تطبيق حديقة السطح له العديد من الدوافع : القيمه البيئية حيث تقلل من الانبعاثات الحراريه وتحد من استخدام المواد

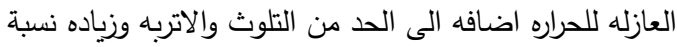
الاوكسجين في الجو، وايضا القيمة الاجتماعية والجماليه، بالاضافة الى القيمه الاقتصاديه حيث تعتبر حديقة السطح

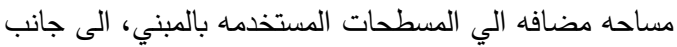
ان تكلفة تشطيب السطح بالطريقة التقليدية(ارضيات سيراميك) تزيد حوالي 50\% عن تكلفه المسطح الاخضر (نجيله). - تبرز مشكلة تطبيق فكره حديقة السطح في العمارات السكنيه لان السطح يكون علي المشاع خاصة في ظل قانون المباني

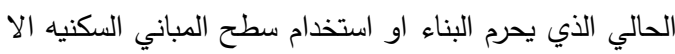

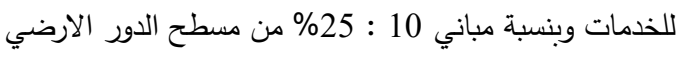

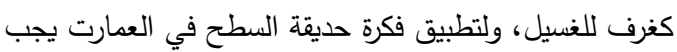
تعديل القانون بحيث يسمح بان يكون السطح وحدة مستقله اوامكانية ان يكون السطح ملحق باحدي وحدات الدور الاخير .

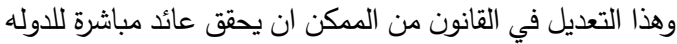

ولكن الهدف الرئيسي هو تحديد هويه المستقيد من السطح. - حديقة السطح في اسطح المباني العامه: تطبيق فكره حديقه

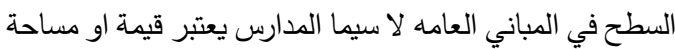
مضافه الي فناء المدرسه خاصة في المدارس التي تم فيها بناء فصول اضافيه في فناء المدرسه.

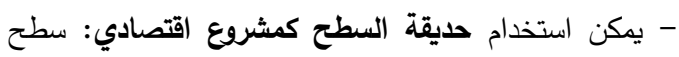

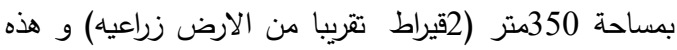
المساحه فى الريف تعتبر مصدر رزق لاسره على مدار العام،

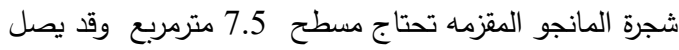
انتاجها الي 150 كجم في السنه كحد اقصي، فاذا كان السطح بمساحة 350مترمربع يستوعب حوالي 45شجرة مانجو مقزمه بانتاج يمكن ان يصل الي 7طن سنويا.
العديد من المسئولين في مصر علاوة على قصر دور الإعلام في الإرشاد والتوجيه. ارتفاع أسعار البذور والثتلات وخاصـة بذور الخضاد وليهر

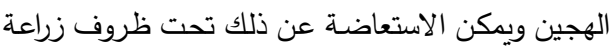
الأسطح ببذور أصناف الخضر في الحقل المكثوف التي قد تكون غير عالية الإنتاج أو ذات جودة أقل ولكنها تفي بالمطلوب منها. عدم توافر الأسمدة أو المحاليل المغذية للمحاصيل المنزرعة فوق الأسطح في المحلات واقتصار توافرها

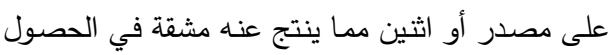
عليها حيث إن جزء كبير من نجاح تلك الزراعة يعود إلى عملية التسميد. عدم وجود خطوط مياة ري في الددن المصرية الجديدة

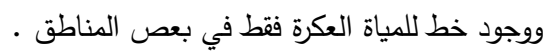

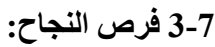
وهي توافر شروط في المكان المخصص لحديقةالسطح: -

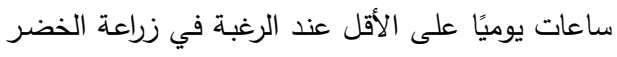

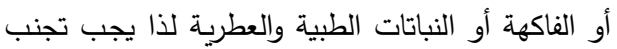

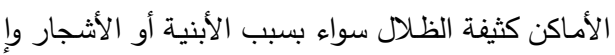
ن لم يمنع ذلك من استخدامها في زراعة نباتات الزينة. تجنب الأماكن المعرضة للرياح الثديدة.

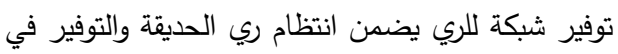

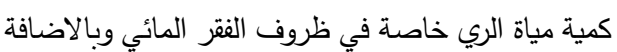
الى ان الري في هذه الحالة لا يشكل عبنًا على القائم

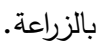

4-7 المخاطرو التهايدات:

عدم الصيانة والمراعاة البيئية يؤدي الى انتثار الحشرات

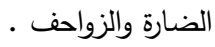
عدم صيانة المبنى قد يؤدي الى اضرار انثائية في حالة

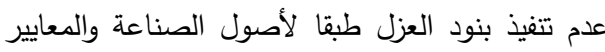
المصرية 


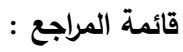 \\ 1-9 المراجع الاجنبية:}

1. Abdel Salam, A., (2009), "The Future of Green-

Roofs in Egypt -The Economical and

Environmental benefits when installing green-

roof on a residential building in Cairo"

Unpublished Msc. Thesis, Architectural

department, the Faculty of Engineering, Cairo

University.

2. Attia, S. \& Mahmoud A., (2009). "Green Roofs in Cairo: A Holistic Approach for Healthy

Productive Cities", In Conference Proceeding on Greening Rooftops for Sustainable

Communities, June 3-5, 2009, Atlanta, USA

3. El Zafarany., (1995)., "Existing Green Areas in Cairo Comparison with Planning Criteria and International Norms." 4th scientific conference, Azhar university.

4. Gawad, I., (2014), The Rise Of Rooftop Gardens In Informally Developed Areas

In Egypt: Exploring The Abilities And Boundaries, 6th International Conference, Architecture department, Faculty of Engineering, Cairo university.

5. Jerry Yudelson, Green Building A to Z, New Society Publishers, 2007

6. Oberndorfer, E., Lundholm, J., Bass B., Coffman R., Doshi H., Dunnett N., Gaffin S., Kohler M., Liu K., Rowe B., (2007), "Green roofs a surban ecosystems: ecological structures, functions, and services", Journal of Bioscience, vol.57, 823-833.

7. Mclntosh, A., (2010), Green Roofs in Seattle, a survey of vegetated roofs and rooftop gardens, the University of Washington.

8. Hebaalla Mostafa, (2016). "Evaluation of Green Building Ratting Systems for Egypt" Msc.

Thesis., Center for Sustainable Development. The American University in Cairo .

9. Timothy Carter, Andrew Keelerb.2008." Lifecycle cost-benefit analysis of extensive vegetated roof systems". Journal of Environmental Management 87 (2008) 350-363
- يجب دراسة اعادة استخدام المياة الناتجة عن الوحدات السكنية • وتصميم شبكات ري او وحدات معالجة للمياة على مستوى( المبنى , الحي , المدينة ) لتحقيق الاستدامة في ظل معاناة مصر من الفقر المائي طبقا لتقارير الحكومة المصرية و الامم المتحدة .

- الاتجاة الى استخدام اساليب الري الحديثة واستخدام تكنولوجيا الري لتحقيق اعلى معدل من الكفاءة مع اقل معدل لاستخدام

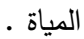

- وضع عوامل تحفيزية لملاك الوحدات واصحاب المنشآت لتشجيعهم على انشاء حدائق اسطح لتحقيق الفوائد البيئية للمجتمع كله . ومثثل هذه الحوافز التي رصدها البحث في التجارب العالمية ( تخفيض فاتورة الكهرباء والمياة للمبنى اعفاء الدور الاخير من فاتورة مياة الري - تخفيض رسوم التراخيص - تخفيض الضريبة العقارية للمبنى ). - زيادة الوعي البيئي والعمراني في المدارس و ادخال مناهج التعليم الاخضر Green Education ( ) لزيادة الوعي الجمعي لدى الاطفال(Fernandez, 2013) بالأضافة الى الاهتمام بالمشاريع التطبيقية الخضراء لطلاب كليات الهندسة.

ادخال دراسات الاثر البيئي للمباني طبقا لأجراءات الترخيص (Hebaalla, في المباني العامة ووضع نظام الهرم الأخضر (2016 جزء من تقييم المبنى قبل مرحلة الترخيص 
مبنى سكنى فى القاهرة، رسالة ماجستير ، كلية الهندسة، جامعة

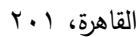

2. مروة الثعار .2017 , "التتمية المستدامة للمجتمعات العمرانية

بأستخدام تقنيات الزراعة الحديثة", رساله ماجيستير , قسم العماره بكليه الفنون الجميله, جامعة حلوان

3. دينا عيد سعيد خاطر، 2014، الأسطح الخضراء في الإسكان-

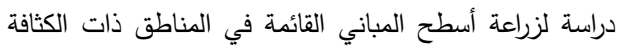

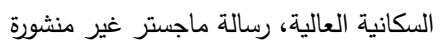

10. Rafael Fernandez-Cañero, Tobias Emilsson ,Carolina Fernandez-Barba ,Miguel Ángel Herrera Machuca. 2013 ." Green roof systems: A study of public attitudes and preferences in southern Spain ". Journal of Environmental Management 128 (2013) 106e115

11. Khan, S., and Asif, M., (2017), Impact of Green Roof and Orientation on the Energy Performance of Buildings: A Case Study from Saudi Arabia,

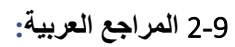

1. أحمد السيد سعد عبد السلام، مستقبل الأسطح الخضراء فى الخى

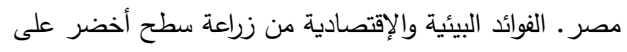




\title{
Using roof garden as a green infrastructure application To achieve sustainability in new cities
}

\begin{abstract}
:
In light of the current climatic changes, many countries are seeking to use Green Infrastructure to integrate with gray infrastructure due to their environmental, social and economic benefits. The green infrastructure networks are distinguished by the diversity of their applications, whether at the level of the building, streets or the urban district, and the integration between these various applications maximizes their environmental impact. The roof gardens represent one of the applications of green infrastructure networks that research focuses on and proposes to apply them on a wider scale in Egypt, because most Egyptian cities suffer from environmental, social and economic stress.

The research aims to clarify the legislative and executive challenges of roof gardens in new cities, in addition to the benefits resulting from designing roof gardens on residential units.

The study deals with using the technology of rooftop garden cultivation in new cities as a means to achieve sustainability. The research presents theoretical studies of roof gardens and an applied study in one of the new cities - Sheikh Zayed City - and the research concludes with an applied methodology for implementing this model in many new cities.
\end{abstract}

Keywords: Roof garden, Sustainability, green infrastructure, new cities 